\title{
The immunomodulatory potential of the arylmethylaminosteroid sc10
}

\author{
Leonard Blum $^{1,2} \cdot$ Thomas Ulshöfer $^{1} \cdot$ Marina Henke ${ }^{1} \cdot$ Reimar Krieg $^{3} \cdot$ Isabell Berneburg $^{3} \cdot$ Gerd Geisslinger $^{1,2}$. \\ Katja Becker ${ }^{4} \cdot$ Michael J. Parnham ${ }^{1}$. Susanne Schiffmann ${ }^{1}$
}

Received: 11 May 2020 / Revised: 18 November 2020 / Accepted: 11 December 2020 / Published online: 17 December 2020

(C) The Author(s) 2020

\begin{abstract}
Developing resistance mechanisms of pathogens against established and frequently used drugs are a growing global health problem. Besides the development of novel drug candidates per se, new approaches to counteract resistance mechanisms are needed. Drug candidates that not only target the pathogens directly but also modify the host immune system might boost antiparasitic defence and facilitate clearance of pathogens. In this study, we investigated whether the novel anti-parasitic steroid compound 1o (sc1o), effective against the parasites Plasmodium falciparum and Schistosoma mansoni, might exhibit immunomodulatory properties. Our results reveal that $50 \mu \mathrm{M}$ sc1o amplified the inflammatory potential of M1 macrophages and shifted M2 macrophages in a pro-inflammatory direction. Since M1 macrophages used predominantly glycolysis as an energy source, it is noteworthy that sc1o increased glycolysis and decreased oxidative phosphorylation in M2 macrophages. The effect of sc1o on the differentiation and activation of dendritic cells was ambiguous, since both pro- and anti-inflammatory markers were regulated. In conclusion, sc 1o has several immunomodulatory effects that could possibly assist the immune system by counteracting the anti-inflammatory immune escape strategy of the parasite $P$. falciparum or by increasing pro-inflammatory mechanisms against pathogens, albeit at a higher concentration than that required for the anti-parasitic effect.
\end{abstract}

\section{Key messages}

- The anti-parasitic steroid compound 1o (sc1o) can modulate human immune cells.

- Sclo amplified the potential of M1 macrophages.

- Sc1o shifts M2 macrophages to a M1 phenotype.

- Dendritic cell differentiation and activation was ambiguously modulated.

- Administration of sclo could possibly assist the anti-parasitic defence.

Keywords Steroid compound 1o $\cdot$ Macrophages $\cdot$ Dendritic cells $\cdot$ Immune modulation $\cdot$ Immune metabolism . Plasmodium falciparum

Susanne Schiffmann

susanne.schiffmann@ime.fraunhofer.de

$1 \quad$ Fraunhofer Institute for Molecular Biology and Applied Ecology IME, Branch for Translational Medicine and Pharmacology (TMP), Theodor-Stern-Kai 7, 60596 Frankfurt am Main, Germany

2 pharmazentrum frankfurt/ZAFES, Department of Clinical Pharmacology, Goethe-University Hospital Frankfurt, Theodor-Stern-Kai 7, 60590 Frankfurt/Main, Germany

3 Department of Anatomy II, University Hospital Jena, Teichgraben 7, 07743 Jena, Germany

4 Biochemistry and Molecular Biology, Interdisciplinary Research Center, Justus-Liebig-University, Heinrich-Buff-Ring 26-32, 35392 Giessen, Germany

\begin{tabular}{ll}
\multicolumn{2}{l}{ Abbreviations } \\
ANOVA & Analysis of variance \\
ECAR & Extracellular acidification rate \\
FCS & Foetal calf serum \\
G M - & Granulocyte macrophage colony-stimulating \\
CSF & factor \\
IFN & Interferon \\
IL & Interleukin \\
MdM & Monocyte-derived macrophage \\
MdDC & Monocyte-derived dendritic cell \\
OCR & Oxygen consumption rate \\
PBS & Phosphate-buffered saline \\
sc1o & Steroid compound 1o
\end{tabular}




\section{Introduction}

Increasingly resistant pathogens are causing a global health problem with a variety of infectious diseases. The most important treatments for parasite-mediated diseases such as malaria (Plasmodium) and schistosomiasis (Schistosoma) are artemisinin combination therapies for malaria and praziquantel for schistosomiasis. The WHO reported 228 million cases of malaria worldwide in 2018 [1], while schistosomiasis affects approximately 200-250 million people, mostly in developing countries [2-4]. The frequent use of the available drugs increases the risk of resistance mechanisms. Reports relating to artemisinin-resistant parasites stress the urgent need for new therapeutic approaches $[5,6]$. Besides resistance mechanisms against the drugs, Plasmodium further accentuates the challenge as it reduces the defensive pro-inflammatory conditions of the host by promoting the M2-phenotype of monocytes, probably through haemozoin-induced CD206 expression [7, 8].

In addition to the direct impact of new drug candidates on pathogens, maintenance and promotion of immune responses are also important to overcome emerging pathogen resistance. Modulating the immune system is a promising approach to boost host defence mechanisms and increases the clearance of pathogens while minimizing tissue damage from inflammation. It has been shown that several antibiotics are able to modulate inflammatory processes and thereby promote pathogen defence even in the face of mechanisms to resist the direct antimicrobial impact of the drugs [9]. Therefore, immunomodulation can expand the efficacy profile and may unfold new therapeutic indications as a pro- or antiinflammatory modulator.

Inflammation accompanies the majority of infections, regardless of whether they are caused by bacteria or parasites, and is characterized by the accumulation of various immune cells such as neutrophils, dendritic cells, monocytes, and macrophages at the site of infection [10]. This can result in the release of a variety of lipid mediators, cytokines, chemokines, growth factors, and enzymes that mediate pathogen killing but can also lead to bystander tissue injuries. In order to prevent an exaggerated immune response and protect the host tissue, a well-timed resolution of the inflammatory process is mandatory. Macrophages are essential for the local initiation of inflammation since they release several cytokines such as interleukin (IL)-1 $\beta$, interferon (IFN)- $\gamma$, IL-23, and tumour necrosis factor (TNF)- $\alpha$. Furthermore, they recruit additional immune cells by secreting chemokines such as CC-chemokine ligand (CCL)2, C-X-C motif chemokine (CXCL)10, and CXCL8 [11]. Besides activating the inflammatory process, macrophages recognize and ingest pathogens and activate $\mathrm{T}$ cells via HLA-DR. During the resolution of inflammation, macrophages release growth factors, cytokines (e.g. IL-10 and IL-4), and chemokines (e.g. CCL18 and CCL17) to recruit anti-inflammatory $\mathrm{T}_{\mathrm{H}} 2$ and $\mathrm{T}_{\text {reg }}$ cells to support the tissue healing process [11-14]. The immune response is regulated not only by macrophages but also by dendritic cells. The expression of specific surface markers such as CD40, CD80, CD86, and HLA-DR is essential for the presentation of antigens and the regulation of the antigen-specific $\mathrm{T}$ cell response by dendritic cells. Since macrophages and dendritic cells are important players in the fight against Plasmodium falciparum [15], we focused on these cell types. In malaria, excessive production of inflammatory cytokines, including TNF- $\alpha$, IL-6, IL-12, and IFN- $\gamma$, at the early stages of infection is a key contributor to pathogenesis [16]. Mouse studies revealed that specifically dendritic cells are responsible for the cytokine release [17]. M1 macrophages form the first line in host defence against parasites. Since macrophages are highly adaptive to their local microenvironment and have been shown to switch their programming from one functional phenotype to another in response to local signals and M2 macrophages are thought to play an important role in maintaining the balance between inflammation and the restoration of tissue homeostasis after infection and local injury, we focused on both phenotypes.

Approved antibiotics with immunomodulatory effects include azithromycin and quinolones. Quinolones such as moxifloxacin interact with the immune response in a biphasic mode [18]. They initially induce the release of proinflammatory cytokines, phagocytosis, and the oxidative burst [19], subsequently leading to a halt in cytokine production. The steroid compound $1 \mathrm{o}$ ( $\mathrm{sc10}$ ), a new lead compound with a promising activity profile against Plasmodium falciparum and Schistosoma mansoni parasites [20] and a good safety profile [21], consists of a steroid and a 2-hydroxyarylmethylamino moiety [20]. The 2-hydroxymethylamino residue is accessible to oxidative transformations that lead to quinone methide intermediates, which have some (formal) structural similarities with quinolones. Therefore, we speculated that sc1o might also interact with the immune system, and in this study, we have investigated some potential immunomodulatory effects of sclo using standard stimuli. With this aim in mind, we tested the impact of sc1o on differentiation and polarization/ activation of human monocyte-derived macrophages (MdM) and dendritic cells (MdDC) in vitro.

\section{Results}

\section{Sc10 did not reduce cell viability in myeloid cells}

Beside the specificity and potency of an antimicrobial drug, another prerequisite is that it is not cytotoxic to myeloid cells. We thus investigated whether sc1o influences cell viability of primary myeloid cells such as MdMs and MdDCs.

Unexpectedly, sc1o increased the metabolic activity of MdMs and MdDCs tested by the Orangu ${ }^{\mathrm{TM}}$ assay, which measures the dehydrogenase activity in living cells using a 
tetrazolium substrate (Fig. 1a and b). Concentrations above $75 \mu \mathrm{M}$ significantly increased metabolic activity in both cell types. The strongest effects could be detected in MdDCs with an increase of up to approximately $400 \%$ at $100 \mu \mathrm{M} \mathrm{sc} 1 \mathrm{o}$. Since higher concentrations are physiologically less relevant, a maximum concentration of $50 \mu \mathrm{M}$ sclo was used in the following experiments to test the immunomodulatory potential.

\section{Sc10 influenced the differentiation of dendritic cells}

To test whether sclo influences the process of dendritic cell differentiation, isolated monocytes were differentiated in the presence of different concentrations of sc1o $(0.5-50 \mu \mathrm{M})$ or vehicle (DMSO). As a readout, surface markers that are regulated during differentiation were determined. Furthermore,
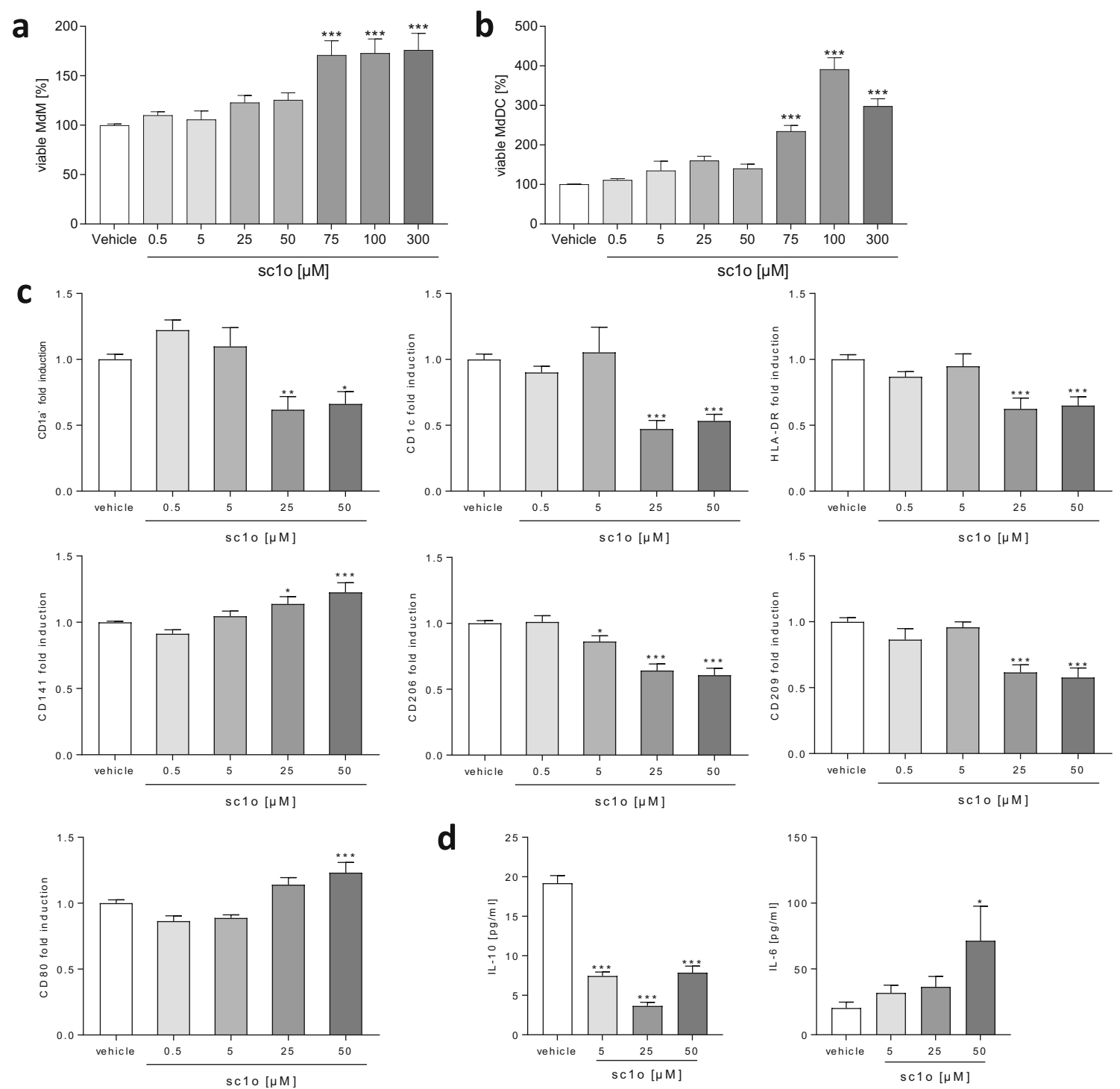

d
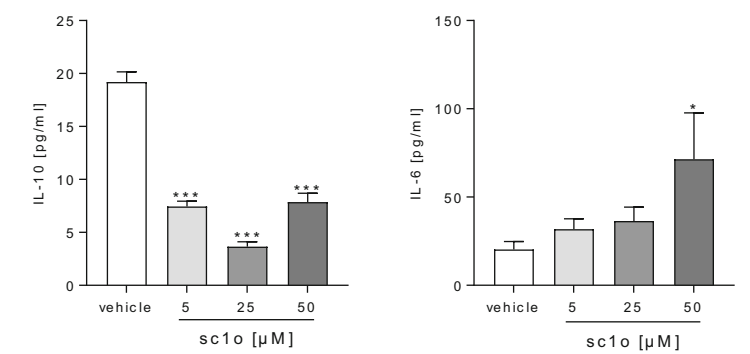

Fig. 1 Sc1o influences myeloid cell viability and dendritic cell differentiation. The percentage of viable human monocyte-derived macrophages $(\mathrm{MdM})(\mathbf{a})$ and monocyte-derived dendritic cells $(\mathrm{MdDC})(\mathbf{b})$ in the presence or absence of different concentrations of sc1o $(0.5-300 \mu \mathrm{M})$ or vehicle (DMSO) were determined with an Orangu ${ }^{\mathrm{TM}}$ assay in triplicate. Therefore, human $\mathrm{CD} 14^{+}$cells were isolated from buffy coats and differentiated to MdMs (7 days, $10 \mathrm{ng} / \mathrm{ml} \mathrm{GM-CSF}$ ) or MdDCs (5 days, $10 \mathrm{ng} / \mathrm{ml} \mathrm{GM-CSF}$ and IL-4). After differentiation, cells were treated with different sc1o concentrations for $48 \mathrm{~h}$. Viability was measured after $120 \mathrm{~min}$ incubation with the Orangu reagent. The percentage values were calculated with vehicle-treated cells as reference $(n(\mathbf{a}, \mathbf{b})=3$ blood donors in 1 experiment). The influence of sc1o on dendritic cells during differentiation (c/d). Human monocytes were isolated and differentiated

to MdDCs for 5 days with GM-CSF (10 ng/ml) and IL-4 (10 ng/ml) in the presence or absence of different concentrations of sc1o $(0.5-50 \mu \mathrm{M})$ or a vehicle (DMSO). (c) Surface marker expression was measured with a MACSQuant ${ }^{\circledR}$ Analyser 10 in triplicate. Fold induction of the geometric mean of the fluorescence intensity was calculated by referring treated cells to vehicle controls $(n=6-11$ different blood donors in 4 separate experiments). (d) Released concentrations of IL- 6 and IL-10 in the supernatant were measured with a cytometric bead array in triplicate $(n=4$ different blood donors in 3 separate experiments). For statistical analysis, a one-way ANOVA with Dunnett's multiple comparisons test (a-d) was used. Results are presented as means \pm standard errors. $* p<0.05$. $* *$ $p<0.01 . * * * p<0.001$ 
released cytokines and chemokines were detected. Our results showed that the incubation with sclo results in pro- and antiinflammatory changes in MdDCs (Fig. 1c-d). The antigenpresenting surface markers (CD1a, CD1c, HLA-DR) and receptors essential for the phagocytosis of pathogens (CD206 and CD209) were downregulated by 25 und $50 \mu \mathrm{M}$ sc1o. However, $50 \mu \mathrm{M}$ sc1o significantly upregulated CD141 and the receptor $\mathrm{CD} 80$, essential for the activation of $\mathrm{T}$ cells, during the differentiation process (Fig. 1c). Sc1o did not regulate CD54, CD40, CD83, CD197, and CD86 (Supplemental Fig. 1a) and decreased the release of IL-10 (Fig. 1d). IL-6 release was increased by $50 \mu \mathrm{M}$ sc1o, whereas IL-23 and IL- 8 were not influenced by sc1o during MdDC differentiation (Fig. 1d, Supplemental Fig. 1b). These data indicate that sclo possibly reduces the potential of MdDC for antigen presentation and phagocytosis.

\section{Inhibition of dendritic cell activation by sc10}

Furthermore, we investigated to what extent sc1o influences the activation of MdDCs. We found that MdDCs treated with sc1o are characterized by a reduced expression of receptors essential for T cell activation (CD80, CD86, CD40, partially even from $5 \mu \mathrm{M}$ ), antigen presentation (CD1c), phagocytosis (CD206, CD209), and the chemokine receptor CD197 (Fig. 2a). On the other hand, HLA-DR, CD83, CD141, CD54, and CD1a were not noticeably modified (Supplemental Fig. 2a). Twenty-five and $50 \mu \mathrm{M}$ sc1o induced the release of IL-23, which provokes, among other effects, the differentiation of $\mathrm{TH}_{17}$ cells [22] (Fig. 2b). However, several other cyto-/chemokines (IL-12p70, IL-10, IL-6, CXCL8) were not significantly influenced by sc1o treatment (Supplemental Fig. 2b). These data indicate that sc1o possibly impairs antigen presentation, phagocytosis, and the $\mathrm{T}$ cellactivating potential of MdDCs.

\section{Sc10 influenced macrophage differentiation}

Besides the process of MdDC differentiation, we examined the influence of sclo on MdM differentiation. Sc1o significantly increased the expression of the phagocytosis receptor CD206 at the lowest tested concentrations and slightly reduced a receptor relevant to antigen presentation (HLA-DR) (Fig. 3a), whereas sc1o did not modify CD80, CD86, CD163, and TREM2 (Supplemental Fig. 3). Sc1o significantly reduced the release of CCL17 and IL-10. CCL18 and IL-6 were significantly increased (Fig. 3b). These data indicate that, during differentiation, sclo directs the macrophages towards an anti-inflammatory state, as reflected by the increase in antiinflammatory marker CD206, and decreases the antigenpresentation marker HLA-DR. However, the environment generated by the sc1o-treated macrophage in releasing cytokines and chemokines is characterized by both anti- and pro-inflammatory influences.

\section{Sc10 promoted the pro-inflammatory potential of polarized macrophages}

Next, we focused on the influence of sc1o on the M1 and M2 polarization of differentiated macrophages. Surface markers regulated during macrophage polarization (e.g. CD80, CD86, CD163, CD206, TREM2, HLA-DR) were determined. In addition, released cytokines and chemokines (e.g. IL-1 $\beta$, IL-6, IL-10, IL-23, IFN- $\gamma$, TNF- $\alpha$, CCL2, CCL18, CXCL8, CXCL10, $\mathrm{PGE}_{2}$ ) were also detected. Sc1o reduced the expression of the anti-inflammatory markers CD206 and TREM2 in M1 macrophages at $50 \mu \mathrm{M}$, whereas CD80, CD86, HLA-DR and CD163 were not modified (Fig. 4a, Supplemental Fig. 4a). Furthermore, $50 \mu \mathrm{M}$ sc1o significantly upregulated the release of the pro-inflammatory mediators TNF- $\alpha$, CXCL8, and IL-23, as well as PGE 2 , whereas CCL2, CCL18, CXCL10, IL-10, and IL-1 $\beta$ were not influenced (Fig. 4b, Supplemental Fig. 4a). In the case of M2 macrophages, sc1o significantly reduced the expression of CD80 and CD206 (the latter already at $10 \mu \mathrm{M}$ ), whereas CD86, CD163, TREM2, and HLA-DR were not changed (Fig. 4c, Supplemental Fig. 4b). Moreover, $50 \mu \mathrm{M}$ sc1o increased the release of the pro-inflammatory TNF- $\alpha$, CCL2, CXCL8, CXCL10, and IL-1 $\beta$ (Fig. 4d). Sc1o did not influence the release of CCL18, IL-23, IL-10, and PGE $_{2}$ (Supplemental Fig. 4b). Most importantly, these data indicate that sc1o drives polarized M1 and M2 macrophages to a more proinflammatory state accompanied by the release of an increased quantity of pro-inflammatory cytokines and chemokines, thereby generating a pro-inflammatory environment.

\section{Sc10 influenced energy metabolism of myeloid cells}

The predominant energy source of M1 macrophages is glycolysis, whereas M2 macrophages use oxidative phosphorylation to meet their energy requirements [23]. Since sc1o increased the viability of MdMs and drives macrophages towards a more pro-inflammatory condition, we investigated whether this was due to modified energy metabolism. For this, OCR, a marker for mitochondrial respiration, and ECAR, a marker for glycolysis, were determined (Fig. 5). In M1-polarized macrophages, sc1o transiently reduced OCR depending on concentration (Fig. 5a), while ECAR was not notably modified. In M2polarized macrophages, $50 \mu \mathrm{M}$ sc1o reduced OCR, while ECAR was significantly increased by 10 and $50 \mu \mathrm{M}$ sc1o (Fig. 5b). Since increasing metabolic rates can be linked to cell death [24], we investigated whether sc1o induces cell death in M1 and M2 MdM cells. However, sc1o reveal no cytotoxic effects in M1 and M2 MdM cells (Fig. 5a, b, extreme right). 
a

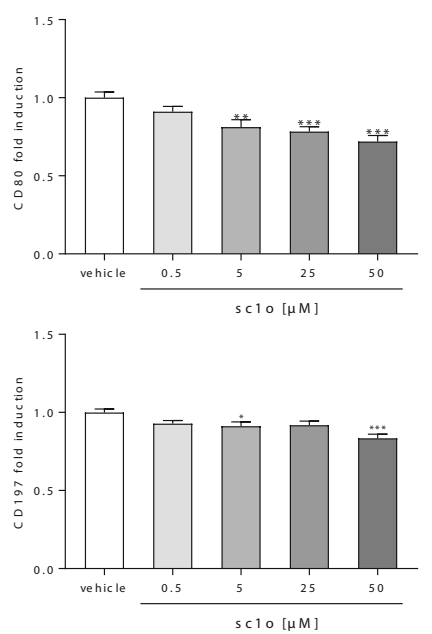

Fig. 2 Effect of sclo on activated MdDCs. Human monocytes were differentiated to MdDCs for 5 days with GM-CSF (10 ng/ml) and IL-4 $(10 \mathrm{ng} / \mathrm{ml})$. MdDCs were activated with a mixture of cytokines (TNF- $\alpha$, IL-6, IL-1 $\beta$ ) and PGE2 in the presence or absence of different concentrations of sc1o $(0.5-50 \mu \mathrm{M})$ or a vehicle (DMSO) for $24 \mathrm{~h}$. (a) Surface marker expression was measured with a MACSQuant ${ }^{\circledR}$ Analyser 10 in triplicate. Fold induction of the geometric mean of the fluorescence
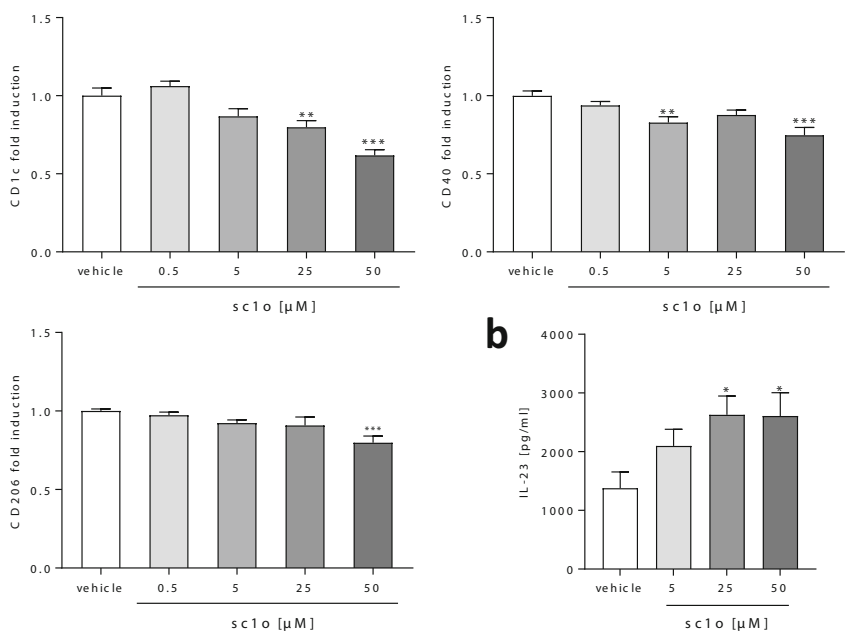

b

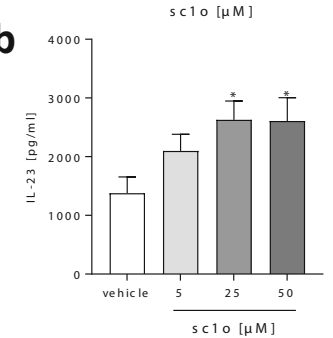

intensity was calculated by referring treated cells to vehicle controls ( $n=6$ different blood donors in 5 separate experiments). (b) Released concentrations of IL-23 in the supernatant were measured with ELISA in triplicate ( $n=6$ different blood donors in 5 separate experiments). For statistical analysis, a one-way ANOVA with Dunnett's multiple comparisons test $(\mathbf{a}-\mathbf{b})$ was used. Results are presented as means \pm standard errors. ${ }^{*} p<0.05$. $* * p<0.01$. ${ }^{* * *} p<0.001$

a

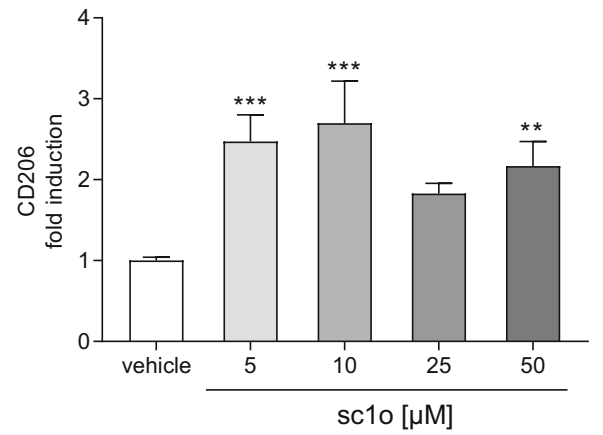

b


Fig. 3 The influence of sc1o on macrophages during differentiation. Human monocytes were isolated and differentiated to MdMs (7 days, $10 \mathrm{ng} / \mathrm{ml} \mathrm{GM-CSF}$ ) in the presence of different concentrations of sc1o $(5-50 \mu \mathrm{M})$ or vehicle (DMSO). (a) Surface marker expression of MdMs was measured with a MACSQuant ${ }^{\circledR}$ Analyser 10 in triplicate. Fold induction of the geometric mean of the fluorescence intensity was calculated by referring treated cells to vehicle controls $(n=6-14$ different blood donors in 7 separate experiments). (b) Released cytokines in the
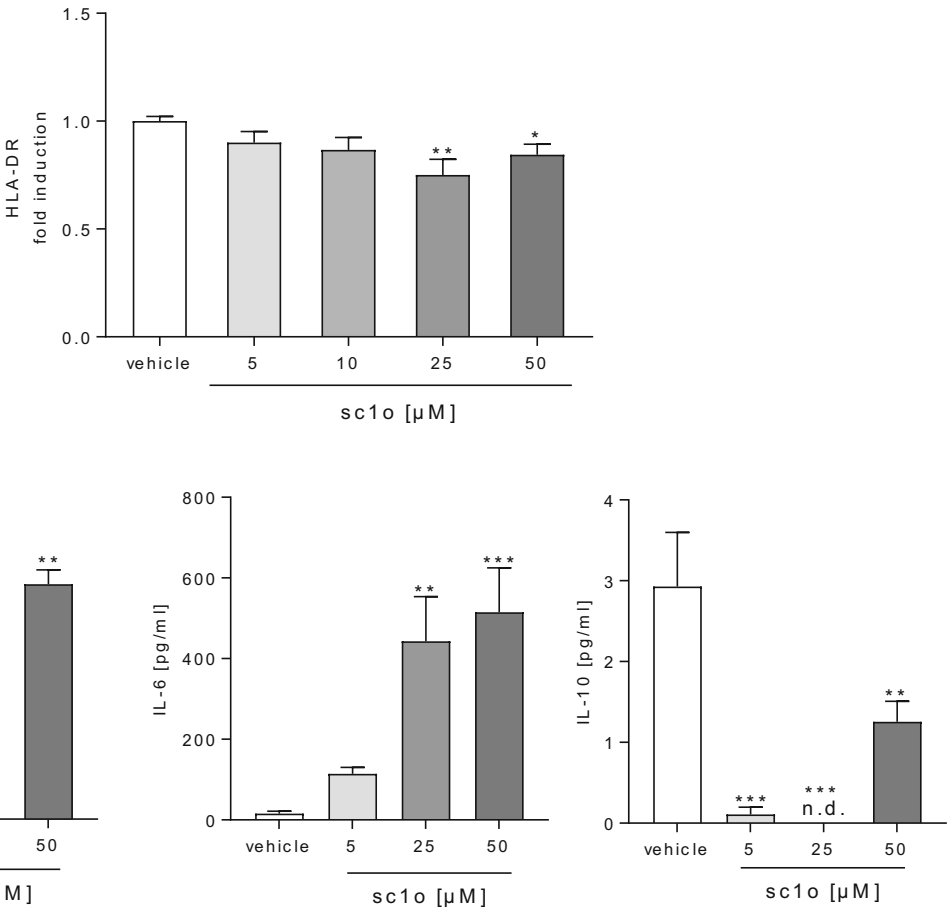

supernatant of MdMs after differentiation with $\operatorname{sc} 1 \mathrm{o}(5-50 \mu \mathrm{M})$ for 7 days. Cytokine concentrations of IL-10/IL-6 were measured with a cytometric bead array and CCL18/CCL17 with ELISA in triplicate $(n=4)$. For statistical analysis, a one-way ANOVA with Dunnett's multiple comparisons test (a-d) was used to compare different sclo concentrations with a vehicle. Results are presented as means \pm standard errors. ${ }^{*} p<0.05$. $* * p<0.01$. ***p $p<0.001$ 



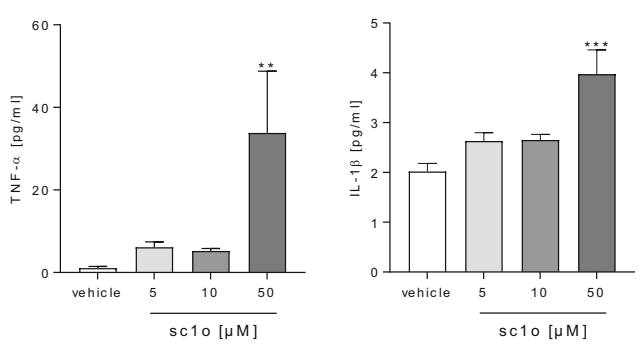

Fig. 4 Influence of sc1o on M1- or M2-polarized macrophages. Human monocytes were isolated from buffy coats and differentiated to M1 monocyte-derived macrophages (M1 MdMs) for 7 days using GM-CSF $(10 \mathrm{ng} / \mathrm{ml})$ or M2 MdMs using M-CSF (50 ng/ml). After differentiation, cells were polarized with IFN- $\gamma(20 \mathrm{ng} / \mathrm{ml})$ or IL-4 $(10 \mathrm{ng} / \mathrm{ml})$ in the presence of different sc1o concentrations $(5-50 \mu \mathrm{M})$ or a vehicle (DMSO) for $48 \mathrm{~h}$ or $24 \mathrm{~h}$ to M1 or M2 macrophages, respectively. Surface marker expression and the cytokine/chemokine level of M1 MdMs (a, b) or M2 MdMs (c, d) are shown. Surface markers were

These data indicate that sc1o increased glycolysis in M2 macrophages, whereas oxidative phosphorylation was suppressed in M1 and M2 macrophages.

\section{Discussion}

Initially, sc1o was identified as a new and promising candidate for the treatment of malaria [20]. Beside its direct antiparasitic effects, we surmised that sclo might have an additional immune modulating character. In the current study, we could show that sc1o up to $300 \mu \mathrm{M}$ did not reduce viability in measured with a MACSQuant ${ }^{\circledR}$ Analyser 10 in triplicate. Fold induction of the geometric mean of the fluorescence intensity was calculated by referring treated cells to vehicle controls ( $n=6$ different blood donors in 3 separate experiments). Released cytokines in the supernatant were determined with either a cytometric bead array or ELISA in triplicate $(n=6$ different blood donors in 3 separate experiments). For statistical analysis, a one-way ANOVA with Dunnett's multiple comparisons test (a-d) was used. Results are presented as means \pm standard errors.* $p<0.05$. $* * * p<0.001$

MdDCs and MdMs. Moreover, $50 \mu \mathrm{M}$ sclo amplified the inflammatory potential of M1 MdMs, directing M2 MdMs towards an M1 phenotype and suppressing the activation of MdDCs, particularly their expression of CD40 and CD80. The monitored effects are summarized in Fig. 6.

We propose that these effects of sclo are linked to metabolic alterations induced by sc1o. Sc1o amplifies the M1 phenotype of M1 MdMs and shifts M2 MdMs to the M1 phenotype. The metabolic pathways in M1 and M2 macrophages are differentially activated [25]. M1 macrophages are characterized by upregulated glycolysis, impairment of oxidative phosphorylation, and disruption of the Krebs 
a
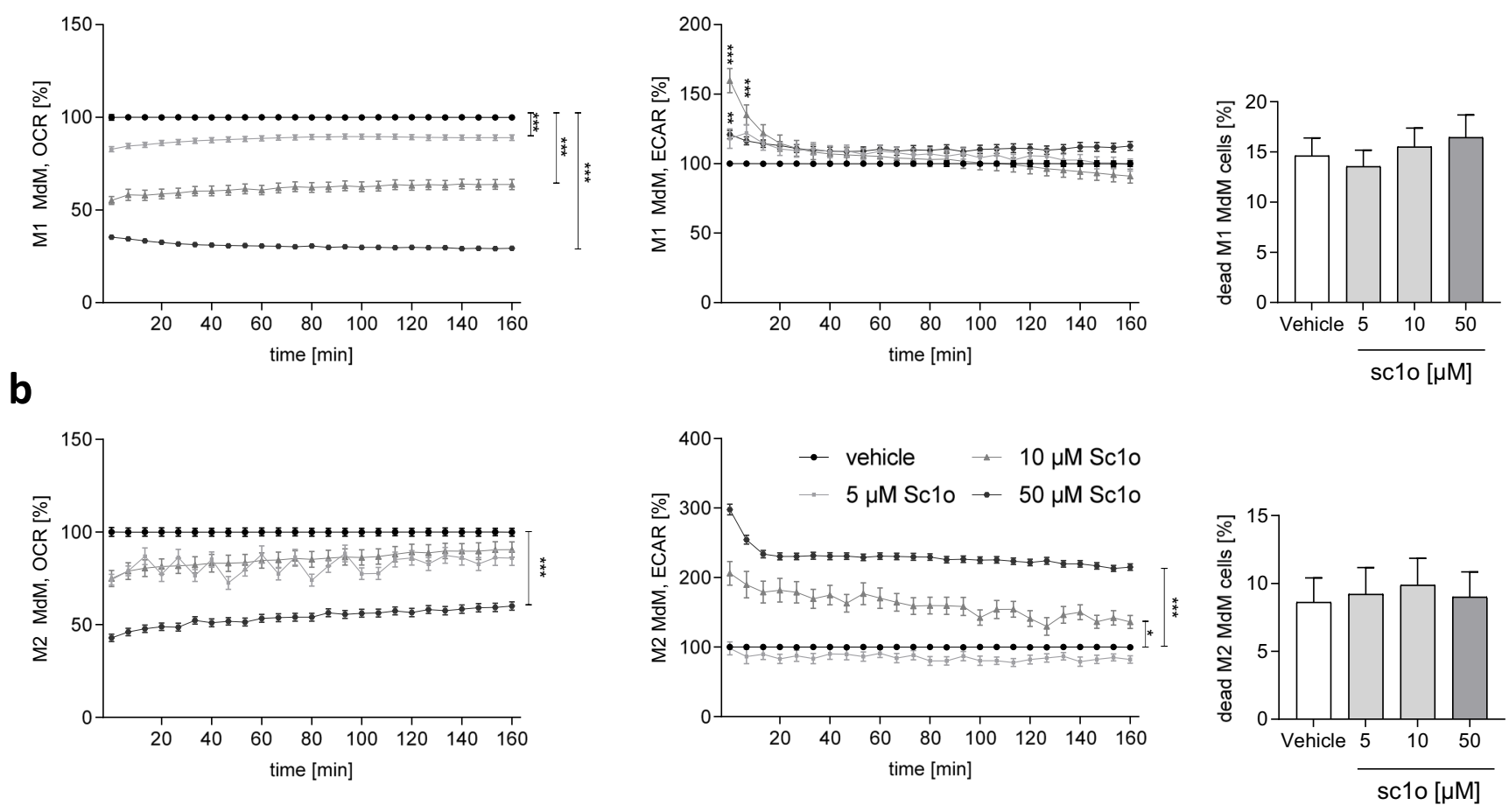

Fig. 5 Sc1o influences the energy metabolism of polarized MdMs. The influence of sclo on the energy metabolism and cell death on polarized M1 MdMs (a) and polarized M2 MdMs (b). Human monocytes were isolated from buffy coats, stimulated (10 ng/ml GM-CSF (M1) or $50 \mathrm{ng} / \mathrm{ml} \mathrm{M}-\mathrm{CSF}$ (M2)), and incubated for 7 days. After differentiation, MdMs were further polarized to M1 and M2 MdMs using IFN- $\gamma$ $(20 \mathrm{ng} / \mathrm{ml})$ or IL-4 $(10 \mathrm{ng} / \mathrm{ml})$ in the presence of 5,10 , or $50 \mu \mathrm{M} \mathrm{sc10}$ for $48 \mathrm{~h}$ or $24 \mathrm{~h}$, respectively. Cell death was determined with the Zombie

Aqua $^{\mathrm{TM}}$ Fixable Viability Kit and analysed by flow cytometry. The extracellular acidification rate (ECAR) and oxygen consumption rate (OCR) were measured with the Seahorse XFe96 analyser (Agilent, Waldbronn, Germany) over a total time period of $160 \mathrm{~min}$. Sc1o-treated cells were compared to vehicle- (DMSO) treated control. To compare ECAR and OCR of sclo-treated cells to vehicle treated control, a twoway ANOVA with Bonferroni's multiple comparisons test was used. Results are presented as means \pm standard errors. $* p<0.05$. *** $p<0.001$

cycle at two steps, after formation of citrate and succinate $[26,27]$. Citrate is used for fatty acid biosynthesis, which allows for the increased synthesis of inflammatory prostaglandins, and for succinate, which activates the transcription factor HIF- $1 \alpha$, a regulator of a wide range of genes, including IL-1 $\beta$, CCL2, and CXCL8 $[26,28,29]$. Therefore, one would expect that sc1o, in shifting M2 MdMs to an M1 phenotype, should be characterized by an increased release of IL-1 $\beta$, CXCL8, and CCL2, by increased glycolysis, and a reduction of oxidative phosphorylation measured. Indeed, we observed such an increased expression of IL-1 $\beta$, CXCL8, and CCL2, an increase in glycolysis (ECAR), and an impairment of oxidative phosphorylation (OCR) in sc1o-treated M2 MdMs. The observed increase in $\mathrm{PGE}_{2}$ levels in sc1o-treated M1 MdMs, in which sc1o amplifies the M1 phenotype, might indicate that the Krebs cycle is disrupted and citrate is used to synthesize $\mathrm{PGE}_{2}$. Additionally, the OCR, which is a metabolic pathway for M2 MdMs, was significantly decreased in M1 MdMs, while ECAR was not affected, probably due to the pre-existing high level of ECAR. These data indicate that

sc1o probably alters the energy metabolism of MdMs, leading to the promotion of an M1-phenotype in M1 and M2 MdMs.

In malaria infections, an imbalance between the M1 and M2 phenotype in monocytes in favour of the M2 phenotype is observed [7]. The strengthening of the M2 phenotype is probably due to haemozoin, a malaria pigment that is involved in the breakdown of human haemoglobin by the parasites and that is linked to numerous immunological effects [8]. The administration of haemozoin increased the expression of the resolutionpromoting surface receptor CD206 as well as the secretion of the cyto- and chemokines IL-10, CCL17, and CCL1 in human monocytes [7]. Furthermore, the M1 phenotype is suppressed due to less NO and ROS formation. The involvement of the signalling pathways, $\mathrm{p} 38$-mitogen-activated protein kinase (p38 MAPK), phosphoinositide-3-kinase (PI3K)/protein kinase $\mathrm{B}$, and NFKB in the enhancement of the M2 phenotype was demonstrated. The M2 phenotype is further characterized by the expression of the surface marker CD163 and the formation of the enzyme arginase 1 . Investigations in children infected with malaria showed increased levels of arginase 1 and IL-10, 



Dendritic Cell

Fig. 6 Overview of sc1o $(50 \mu \mathrm{M})$-mediated effects on myeloid cells. The effects of sc1o $(50 \mu \mathrm{M})$ during the differentiation of monocytes to macrophages $(\mathrm{MdM})$ and dendritic cells $(\mathrm{MdDC})$, during the polarization of macrophages to M1 or M2 MdM, and during the activation of monocytederived dendritic cells (MdDC) are shown. Only effects with an alteration of at least $20 \%$ are shown. Predominant pro-inflammatory cytokines/ chemokines are labelled in bright red; predominant anti-inflammatory cytokines/chemokines are shown in bright green; and cytokines/

reduced amounts of $\mathrm{NO}$, and increased expression of M2 markers (CD163, CD206) [30]. The malaria drugs chloroquine and artemisinin reduced the haemozoin-induced M2 phenotype by reducing IL-10 and CD206 [7]. By strengthening the M1 phenotype and weakening the M2 phenotype of macrophages by steroid substance 10 , this could counteract the effects of haemozoin and improve the course of malaria, similar to chloroquine and artemisinin. However, it should be remembered that increased inflammation in malaria could also lead to negative effects such as cerebral malaria. This is usually treated with anti-inflammatory drugs such as statins [31]. A strengthening of the pro-inflammatory aspects by sclo could therefore counteract this and prohibit the administration of sc1o. Further studies on the immune modulatory effects of sclo in the presence of $P$. falciparum are needed to clarify these issues.

The increased release of TNF- $\alpha$, IL-23, CCL2, CXCL8, and CXCL10 by sc1o-treated MdMs may contribute to a pro-inflammatory environment at the infection site and thereby promote the immune response against pathogens via several mechanisms. With an amplified release of IL-23, sc1o could induce the formation of $\mathrm{TH}_{17}$ cells, which play a crucial role in host defence against a variety of pathogens, including chemokines with pro- and anti-inflammatory potential are shown in bright blue. The directions of the arrows indicate a decrease $(\downarrow)$ or increase $(\uparrow)$. For a better overview and since the regulation of the surface marker was not as strong as the cytokine/chemokine alteration, the changes in the surface marker are shown as tendencies. Therefore, if the majority of the surface markers point to pro-inflammatory regulation, a pink arrow is shown; if the majority point to an anti-inflammatory regulation, a green arrow is shown; and if they were equal, both arrows are shown bacteria and viruses [32]. The presence of $\mathrm{TH}_{17}$ cells was mainly observed in the liver during the acute erythrocytic stage of Plasmodium chabaudi infection. However, IL-17Adeficient mice showed no significant alterations in the course of Plasmodium chabaudi infection. Therefore, despite activation, $\mathrm{TH}_{17}$ cells have so far not been demonstrated to exert a defined role during Plasmodium infections [33]. Consequently, as sc1o also tended to decrease dendritic cell potential for antigen presentation to $\mathrm{T}$ cells, this would not be of much relevance in a malaria-infected host, except for possible effects on $\mathrm{T}$ cell activation with long-term treatment.

The immune modulatory effects of sclo were observed at concentrations of 25 or $50 \mu \mathrm{M}$. However, previous studies showed a low permeability of sc1o in the Caco- 2 cell barrier assay indicating that only a limited amount of sclo passes through the cell barrier, and possibly also only a small amount of sc1o is absorbed by the cells [21]. In vivo studies revealed a plasma level of $2 \mu \mathrm{M}$ after i.p. dosing at $100 \mathrm{mg} / \mathrm{kg}$ [20]. One possibility to increase the bioavailability of sclo is to use a suitable formulation. Interestingly, pharmacokinetic studies with formulated sclo revealed an increase in plasma levels in comparison to sc1o without any carrier excipients 
(unpublished data). Unfortunately, the formulation only increases the concentration of compounds in vivo not in in vitro cell culture. Thus, further studies are needed to investigate whether the formulated sclo shows immune modulatory potency in vivo.

Taken together, the modulation of MdMs towards a proinflammatory phenotype by sc1o might be an efficient way to counteract the capacity of $P$. falciparum to escape immune clearance mechanisms. Promoting host defence mechanisms might be particularly efficient in the early stages of the disease to minimize the later risk of enhanced cerebral malaria due to inflammatory processes. However, the sclo concentrations needed for anti-parasitic effects are lower than those for the immune modulatory effects. Therefore, if the immune modulatory potential of sclo is likely to be therapeutically useful, as in counteracting the immunosuppressing effects of $P$. falciparum or in parasite resistance, the sclo dosages will need adjusting.

\section{Materials and methods}

\section{Cells and reagents}

Primary human monocytes, macrophages, and dendritic cells were cultured in RPMI1640 GlutaMAX medium supplemented with $10 \%$ FCS. All media contain $1 \%$ penicillin/streptomycin, and the cells were cultured at $37{ }^{\circ} \mathrm{C}$ in a $5 \% \mathrm{CO}_{2}$ atmosphere. Sc1o was dissolved in DMSO and further diluted in media $\left(c_{\text {stock }}=25 \mathrm{mM}\right.$, maximal DMSO concentration during experiments $0.3 \% \mathrm{v} / \mathrm{v}$ EDTA was from Sigma Aldrich (Schnelldorf, Germany). Human FcR blocking reagent, bovine serum albumin, human CD14 MicroBeads, human GM-CSF, human M-CSF, IL-4, and all antibodies for surface staining except CD197 from BioLegend (Fell, Germany) were from Miltenyi Biotec (Bergisch Gladbach, Germany). Human IL-6, IFN- $\gamma$, IL- $1 \beta$, and TNF- $\alpha$ were from PeproTech (Hamburg, Germany). $\mathrm{PGE}_{2}$ and Accutase solution were from Merck (Darmstadt, Germany).

\section{Cell viability assay}

To determine the cell viability of human MdMs and MdDCs, the Orangu ${ }^{\mathrm{TM}}$ assay was used. After isolation of $\mathrm{CD} 4^{+}$cells, MdMs and MdDCs were differentiated as described in the following sections. After differentiation, $1 \times 10^{5}$ cells were seeded in triplicate in 96-well plates. Different concentrations of sclo $(0.5-300 \mu \mathrm{M})$ or vehicle (DMSO) were added, and cells were incubated at $37{ }^{\circ} \mathrm{C}$ and $5 \% \mathrm{CO}_{2}$. After $48 \mathrm{~h}$ of incubation at $37{ }^{\circ} \mathrm{C}$ and $5 \% \mathrm{CO}_{2}, 10 \mu$ of Orangu ${ }^{\mathrm{TM}}$ cell counting solution (Cell Guidance Systems, Cambridge, UK) was added and incubated for $120 \mathrm{~min}$ at $37{ }^{\circ} \mathrm{C}$ and $5 \% \mathrm{CO}_{2}$. After incubation, absorbance was measured at a wavelength of
$450 \mathrm{~nm}$ with a reference at $650 \mathrm{~nm}$ at an EnSpire ${ }^{\circledR} 2300$ Multimode Plate Reader (Perkin Elmer, Lübeck, Germany). To calculate cell viability in the Orangu ${ }^{\mathrm{TM}}$ assay, the absorbance of vehicle-treated cells was set to $100 \%$, and the sc1otreated samples were correlated to them.

\section{Isolation of human $\mathrm{CD}^{+} 4^{+}$cells}

Human peripheral blood mononuclear cells were isolated from buffy coats using density gradient. For this, $25 \mathrm{ml}$ of blood from healthy donors (German Red Cross, Frankfurt, Germany) were mixed with the same amount of Hank's balanced salt solution (Thermo Fisher Scientific, Oberhausen, Germany) and were layered over $15 \mathrm{ml}$ of Biocoll (Merck, Darmstadt, Germany) in Sep-Mate ${ }^{\mathrm{TM}}-50$ tubes (Stemcell Technologies, Cologne, Germany). Tubes were centrifuged (1200 g, $10 \mathrm{~min}, \mathrm{RT}$ ), and human peripheral blood mononuclear cells were isolated from the interphase, washed with $2 \mathrm{mM}$ EDTA/PBS four times and counted using a MACSQuant® Analyser 10 flow cytometer (Miltenyi Biotec, Bergisch Gladbach, Germany). Defined amounts of cells were dissolved in $0.5 \%$ bovine serum albumin $/ 2 \mathrm{mM}$ EDTA/PBS and incubated with $25 \%$ (v/v) human CD14 MicroBeads for $15 \mathrm{~min}$ at $4{ }^{\circ} \mathrm{C}$. Afterwards magnetically labelled cells were separated via magnetic cell separation with LS columns (Miltenyi Biotec, Bergisch Gladbach, Germany) according to the manufacturer's protocol. Cell count was determined with flow cytometry.

\section{Differentiation of human macrophages and dendritic cells}

For differentiation of human MdM or MdDC, $0.5 \times 10^{6}$ or $0.9 \times 10^{6} \mathrm{CD} 4^{+}$cells/well, respectively, were cultivated in 48-well plates in the presence of different concentrations of sc1o $(0.5-50 \mu \mathrm{M})$ or vehicle (DMSO) in triplicate. After $30 \mathrm{~min}$ of pre-incubation for the differentiation of MdM, $10 \mathrm{ng} / \mathrm{ml}$ of human GM-CSF was added, and for MdDC, $10 \mathrm{ng} / \mathrm{ml}$ human GM-CSF/10 ng/ml human IL-4 were added. MdMs were differentiated for 7 days, while MdDCs were incubated for 5 days. Medium including sclo and growth factors was completely changed for MdM after 3 days. After incubation, cells were centrifuged ( $300 \mathrm{~g}, 5 \mathrm{~min}, \mathrm{RT}$ ), and supernatant was stored at $-80^{\circ} \mathrm{C}$ for ELISA and a cytometric bead array. Cells were washed with PBS and harvested by using Accutase $\left(15 \mathrm{~min}, 37^{\circ} \mathrm{C}, 5 \% \mathrm{CO}_{2}\right)$. Cell count was determined with flow cytometry.

\section{Polarization of human macrophages}

For polarization, $7.5 \times 10^{6}$ human $\mathrm{CD} 14^{+}$cells were seeded in T-75 flasks (Thermo Fisher Scientific, Oberhausen, Germany). For subsequent M1 polarization, $10 \mathrm{ng} / \mathrm{ml}$ of 
human GM-CSF was added, while $50 \mathrm{ng} / \mathrm{ml}$ of human MCSF was used for subsequent M2 polarization. During this first differentiation, no sclo was added to the cells to provide an unmodified process. After 7 days, cells were washed and incubated with $5 \mathrm{ml}$ of Accutase $\left(15 \mathrm{~min}, 37{ }^{\circ} \mathrm{C}, 5 \% \mathrm{CO}_{2}\right)$. Afterwards $15 \mathrm{ml}$ medium was added, and cells were scraped off. Cell count was determined using flow cytometry. For polarization, $5 \times 10^{5}$ cells were seeded in triplicate in 48well plates, and sc1o $(5-50 \mu \mathrm{M})$ or vehicle (DMSO) was added. After $30 \mathrm{~min}$ of pre-incubation $\left(37{ }^{\circ} \mathrm{C}, 5 \% \mathrm{CO}_{2}\right)$, $20 \mathrm{ng} / \mathrm{ml}$ of human IFN- $\gamma$ (M1) or $10 \mathrm{ng} / \mathrm{ml}$ of human IL-4 (M2) was added for polarization, respectively. Cells were harvested after $24 \mathrm{~h}(\mathrm{M} 2)$ or $48 \mathrm{~h}$ (M1) of incubation $\left(37^{\circ} \mathrm{C}, 5 \%\right.$ $\mathrm{CO}_{2}$ ). The quantity of dead cells was calculated using the Zombie Aqua ${ }^{\mathrm{TM}}$ Fixable Viability Kit (BioLegend, Fell, Germany).

\section{Activation of human dendritic cells}

To study the activation of dendritic cells, other maturing conditions were selected than for the study of their differentiation. For differentiating monocytes to dendritic cells, $1.5 \times 10^{7}$ isolated $\mathrm{CD} 14^{+}$cells were seeded in T-75 flasks with $50 \mathrm{ng} / \mathrm{ml}$ of human GM-CSF and $50 \mathrm{ng} / \mathrm{ml}$ of human IL-4. After 5 days of differentiation without sc1o, cells were harvested and seeded in triplicate, with $0.9 \times 10^{6}$ cells/well in 48 -well plates. Sc1o $(0.5-50 \mu \mathrm{M})$ or vehicle (DMSO) was added, and after $30 \mathrm{~min}$ of pre-incubation $\left(37^{\circ} \mathrm{C}, 5 \% \mathrm{CO}_{2}\right), 5 \mathrm{ng} / \mathrm{ml}$ human TNF- $\alpha$, $5 \mathrm{ng} / \mathrm{ml}$ human IL-6, $5 \mathrm{ng} / \mathrm{ml}$ human IL- $1 \beta$, and $500 \mathrm{ng} / \mathrm{ml}$ PGE2 were added. Cells were incubated for $24 \mathrm{~h}\left(37^{\circ} \mathrm{C}, 5 \%\right.$ $\mathrm{CO}_{2}$ ) and harvested for analysis via flow cytometry.

\section{Flow cytometry}

For flow cytometry, $1.5-2 \times 10^{5}$ cells of each sample were analysed. All steps were performed on ice if not stated otherwise. First, non-specific antibody binding to Fc- $\gamma$ receptors was blocked with human FcR blocking reagent for $15 \mathrm{~min}$ at $4{ }^{\circ} \mathrm{C}$. For discriminating living and dead cells, the Zombie Aqua $^{\mathrm{TM}}$ fixable viability kit (1:500 dilution, BioLegend, San Diego, CA, USA) was used according to the manufacturer's protocol. After viability, samples were stained with a cocktail of different surface marker antibodies (for MdM, CD14, CD80, CD86, CD163, CD206, TREM2, HLA-DR and for MdDC, CD11c, CD54, CD1a, CD1c, HLA-DR, CD40, CD83, CD141, CD197, CD206, CD209, CD80, CD86) for $15 \mathrm{~min}$ at $4{ }^{\circ} \mathrm{C}$. Afterwards, $250 \mu \mathrm{l}$ of $10 \% \mathrm{FCS} / \mathrm{PBS}$ was added, and cells were centrifuged $\left(300 \mathrm{~g}, 5 \mathrm{~min}, 4^{\circ} \mathrm{C}\right)$. Cells were suspended in $100 \mu \mathrm{l}$ PBS and measured with a MACSQuant ${ }^{\circledR}$ Analyser 10 flow cytometer. The geometric mean of the fluorescence intensity was calculated using FlowJo software v10 (Treestar, Ashland, TN, USA). Fold induction was calculated by referring treated cells to DMSO controls. Exemplary gating strategy was displayed in Supplement Fig. 5.

\section{Determination of chemo- and cytokines in the supernatant}

For detecting chemo- and cytokines in the supernatant of differentiated, polarized, or activated MdM and MdDC, the cytometric bead array (BD Bioscience, Heidelberg, Germany) was used for TNF- $\alpha$, IL-1 $\beta$, IL-6, IL-10, CCL2, CXCL8, and CXCL10, while ELISAs were used to detect IL-23 (Thermo Fisher, Waltham, MA, USA), CCL17 (BioLegend, Fell, Germany), CCL18 (Boster Biological Technology, Pleasanton, CA, USA), and PGE2 (Enzo, Lörrach, Germany). Cytometric bead array was performed according to the manufacturer's protocol. In a deviation from protocol, only half of the capture and detection beads and $25 \mu \mathrm{l}$ of the supernatant were used. ELISAs were performed strictly according to the manufacturer's protocol. For the ELISAs, $100 \mu \mathrm{l}$ of the supernatant was used.

\section{Determination of cell energy metabolism}

To analyse the extracellular acidification rate (ECAR) and oxygen consumption rate (OCR) of polarized macrophages, the Seahorse XFe96 FluxPak (Agilent, Waldbronn, Germany) was used as recommended by the manufacturer. $\mathrm{CD} 14^{+}$cells were isolated, and macrophages were polarized as previously described. Macrophages were polarized in the presence of 5, 10 , or $50 \mu \mathrm{M}$ sc1o. After polarization, cells were washed with Seahorse XF RPMI medium pH 7.4 (Agilent, Waldbronn) and incubated for $60 \mathrm{~min}$ at $37^{\circ} \mathrm{C}$. OCR and ECAR were measured for a total period of $160 \mathrm{~min}$ in the absence of sc1o. Cells were measured as octuplicates with $3 \times 10^{4}$ cells per well with the Seahorse XFe96 Analyser (Agilent, Waldbronn, Germany) and analysed with Wave Software (Agilent, Waldbronn, Germany). Values of vehicle (DMSO)-treated cells were set to $100 \%$ and sc1o-treated cells referred to this.

\section{Statistics}

Results are presented as means \pm standard errors. The data was analysed with one-way or two-way ANOVA and with Dunnett's comparison test. For all calculations and creation of graphs, GraphPad Prism 8 was used, and $p<0.05$ was considered the threshold for significance.

Supplementary Information The online version contains supplementary material available at https://doi.org/10.1007/s00109-020-02024-4.

Authors' contribution All authors contributed to the study conception and design. Material preparation, data collection, and analysis were performed by Leonard Blum, Marina Henke, Thomas Ulshöfer, Isabell Berneburg, and Reimar Krieg. The first draft of the manuscript was 
written by Leonard Blum, Susanne Schiffmann, Katja Becker, Gerd Geisslinger, and Michael Parnham, and all authors commented on previous versions of the manuscript. All authors read and approved the final manuscript.

Funding Open Access funding enabled and organized by Projekt DEAL. This work was supported by the Landesoffensive zur Entwicklung wissenschaftlich-ökonomischer Exzellenz. (LOEWE), Centre Translationale Medizin und Pharmakologie (TMP), the LOEWE Centre Novel Drug Targets Against Poverty-Related and Neglected Tropical Infectious Diseases (DRUID), the Fraunhofer Cluster of Excellence for Immune-Mediated Diseases (CIMD), and the LOEWE Centre Translational Biodiversity Genomics (TBG).

Data availability Data are available in request to corresponding author.

\section{Compliance with ethical standards}

Conflict of interest The authors declare that they have no conflict of interest.

Ethical approval Not applicable.

Open Access This article is licensed under a Creative Commons Attribution 4.0 International License, which permits use, sharing, adaptation, distribution and reproduction in any medium or format, as long as you give appropriate credit to the original author(s) and the source, provide a link to the Creative Commons licence, and indicate if changes were made. The images or other third party material in this article are included in the article's Creative Commons licence, unless indicated otherwise in a credit line to the material. If material is not included in the article's Creative Commons licence and your intended use is not permitted by statutory regulation or exceeds the permitted use, you will need to obtain permission directly from the copyright holder. To view a copy of this licence, visit http://creativecommons.org/licenses/by/4.0/.

\section{References}

1. WHO (2018) World malaria report 2018. World Health Organization, Geneva

2. Steinmann P, Keiser J, Bos R, Tanner M, Utzinger J (2006) Schistosomiasis and water resources development: systematic review, meta-analysis, and estimates of people at risk. Lancet Infect Dis 6(7):411-425

3. WHO (2015) Schistosomiasis. Fact sheet $N^{\circ} 115$ (2015). Geneva: World Health Organization

4. Anisuzzaman, Tsuji N (2020) Schistosomiasis and hookworm infection in humans: disease burden, pathobiology and anthelmintic vaccines. Parasitol Int:102051. https://doi.org/10.1016/j.parint. 2020.102051

5. Sinha S, Medhi B, Sehgal R (2014) Challenges of drug-resistant malaria. Parasite 21:61

6. Wells TN, Hooft van Huijsduijnen R, Van Voorhis WC (2015) Malaria medicines: a glass half full? Nat Rev Drug Discov 14(6): 424-442

7. Bobade D, Khandare AV, Deval M, Shastry P, Deshpande P (2019) Hemozoin-induced activation of human monocytes toward M2-like phenotype is partially reversed by antimalarial drugs-chloroquine and artemisinin. Microbiologyopen 8(3):e00651

8. Esposito A, Tiffert T, Mauritz JM, Schlachter S, Bannister LH, Kaminski CF, Lew VL (2008) FRET imaging of hemoglobin concentration in Plasmodium falciparum-infected red cells. PLoS One 3(11):e3780

9. Parnham MJ (2005) Immunomodulatory effects of antimicrobials in the therapy of respiratory tract infections. Curr Opin Infect Dis 18(2):125-131

10. Wood PJ (2012) Immunological response to infection: inflammatory and adaptive immune responses. Anaesthesia and Intensive Care Medicine 13(6):269-272

11. Atri C, Guerfali FZ, Laouini D (2018) Role of human macrophage polarization in inflammation during infectious diseases. Int J Mol Sci 19(6). https://doi.org/10.3390/ijms19061801

12. Savill J (2000) Apoptosis in resolution of inflammation. Kidney Blood Press Res 23(3-5):173-174

13. Solari R, Pease JE (2015) Targeting chemokine receptors in disease-a case study of CCR4. Eur J Pharmacol 763(Pt B):169-177

14. Chenivesse C, Tsicopoulos A (2018) CCL18 - beyond chemotaxis. Cytokine 109:52-56

15. Ozarslan N, Robinson JF, Gaw SL (2019) Circulating monocytes, tissue macrophages, and malaria. J Trop Med 2019:37208383720839

16. Schofield L, Grau GE (2005) Immunological processes in malaria pathogenesis. Nat Rev Immunol 5(9):722-735

17. Wu X, Gowda NM, Gowda DC (2015) Phagosomal acidification prevents macrophage inflammatory cytokine production to malaria, and dendritic cells are the major source at the early stages of infection: implication for malaria protective immunity development. J Biol Chem 290(38):23135-23147

18. Hall IH, Schwab UE, Ward ES, Ives TJ (2003) Effects of moxifloxacin in zymogen A or S. aureus stimulated human THP1 monocytes on the inflammatory process and the spread of infection. Life Sci 73(21):2675-2685

19. Labro MT (2000) Interference of antibacterial agents with phagocyte functions: immunomodulation or "immuno-fairy tales"? Clin Microbiol Rev 13(4):615-650

20. Krieg R, Jortzik E, Goetz AA, Blandin S, Wittlin S, Elhabiri M, Rahbari M, Nuryyeva S, Voigt K, Dahse HM, Brakhage A, Beckmann S, Quack T, Grevelding CG, Pinkerton AB, Schonecker B, Burrows J, Davioud-Charvet E, Rahlfs S, Becker K (2017) Arylmethylamino steroids as antiparasitic agents. Nat Commun 8:14478

21. Blum L, Gul S, Ulshofer T, Henke M, Krieg R, Berneburg I, Thomas D, Trautmann S, Kurz J, Geyer J, Geisslinger G, Becker K, Parnham MJ, Schiffmann S (2020) In-vitro safety and off-target profile of the anti-parasitic arylmethylaminosteroid 1o. Sci Rep 10(1):7534

22. Iwakura $Y$, Ishigame $H$ (2006) The IL-23/IL-17 axis in inflammation. J Clin Invest 116(5):1218-1222

23. Kelly B, O'Neill LA (2015) Metabolic reprogramming in macrophages and dendritic cells in innate immunity. Cell Res 25(7):771784

24. Green DR, Galluzzi L, Kroemer G (2014) Cell biology. Metabolic control of cell death. Science 345(6203): 1250256

25. Netea MG, Joosten LA, Latz E, Mills KH, Natoli G, Stunnenberg HG, O'Neill LA, Xavier RJ (2016) Trained immunity: a program of innate immune memory in health and disease. Science 352(6284): aaf1098

26. Tannahill GM, Curtis AM, Adamik J, Palsson-McDermott EM, McGettrick AF, Goel G, Frezza C, Bernard NJ, Kelly B, Foley NH, Zheng L, Gardet A, Tong Z, Jany SS, Corr SC, Haneklaus M, Caffrey BE, Pierce K, Walmsley S, Beasley FC, Cummins E, Nizet V, Whyte M, Taylor CT, Lin H, Masters SL, Gottlieb E, Kelly VP, Clish C, Auron PE, Xavier RJ, O'Neill LA (2013) Succinate is an inflammatory signal that induces IL-1beta through HIF-1alpha. Nature 496(7444):238-242

27. Jha AK, Huang SC, Sergushichev A, Lampropoulou V, Ivanova $Y$, Loginicheva E, Chmielewski K, Stewart KM, Ashall J, Everts B, 
Pearce EJ, Driggers EM, Artyomov MN (2015) Network integration of parallel metabolic and transcriptional data reveals metabolic modules that regulate macrophage polarization. Immunity 42(3): 419-430

28. Baay-Guzman GJ, Bebenek IG, Zeidler M, Hernandez-Pando R, Vega MI, Garcia-Zepeda EA, Antonio-Andres G, Bonavida B, Riedl M, Kleerup E, Tashkin DP, Hankinson O, Huerta-Yepez S (2012) HIF-1 expression is associated with CCL2 chemokine expression in airway inflammatory cells: implications in allergic airway inflammation. Respir Res 13:60

29. Cane G, Ginouves A, Marchetti S, Busca R, Pouyssegur J, Berra E, Hofman P, Vouret-Craviari V (2010) HIF-1alpha mediates the induction of IL-8 and VEGF expression on infection with Afa/Dr diffusely adhering E. coli and promotes EMT-like behaviour. Cell Microbiol 12(5):640-653

30. Weinberg JB, Volkheimer AD, Rubach MP, Florence SM, Mukemba JP, Kalingonji AR, Langelier C, Chen Y, Bush M, Yeo TW, Granger DL, Anstey NM, Mwaikambo ED (2016) Monocyte polarization in children with falciparum malaria: relationship to nitric oxide insufficiency and disease severity. Sci Rep 6:29151

31. Reis PA, Estato V, da Silva TI, d'Avila JC, Siqueira LD, Assis EF, Bozza PT, Bozza FA, Tibirica EV, Zimmerman GA, Castro-FariaNeto HC (2012) Statins decrease neuroinflammation and prevent cognitive impairment after cerebral malaria. PLoS Pathog 8(12): e1003099. https://doi.org/10.1371/journal.ppat.1003099

32. Li Y, Wei C, Xu H, Jia J, Wei Z, Guo R, Jia Y, Wu Y, Li Y, Qi X, Li Z, Gao X (2018) The immunoregulation of Th17 in host against intracellular bacterial infection. Mediat Inflamm 2018:65872966587213

33. Mastelic B, do Rosario AP, Veldhoen M, Renauld JC, Jarra W, Sponaas AM, Roetynck S, Stockinger B, Langhorne J (2012) IL22 protects against liver pathology and lethality of an experimental blood-stage malaria infection. Front Immunol 3:85

Publisher's note Springer Nature remains neutral with regard to jurisdictional claims in published maps and institutional affiliations. 